\title{
A High-Yield Area-Power Efficient DWT Hardware for Implantable Neural Interface Applications
}

\author{
Awais M. Kamboh, Andrew Mason and Karim Oweiss
}

\begin{abstract}
Temporal processing of neural recordings with high-density microelectrode arrays implanted in the cortex is highly desired to alleviate the data telemetry bottleneck. By exploiting the energy compactness capabilities of the discrete wavelet transform (DWT), our previous work has shown that it is a viable data compression tool that faithfully preserves the information in the neural data. This paper describes an areapower minimized hardware implementation of the multi-level, multi-channel DWT. Performance tradeoffs and key design decisions for implantable applications are analyzed. A 32channel, 4-level version of the circuit has been custom designed in $0.18 \mu \mathrm{m}$ CMOS and occupies only $0.16 \mathrm{~mm}^{2}$, making it very suitable for high-yield intra-cortical neural interface applications.
\end{abstract}

\section{INTRODUCTION}

$\mathrm{N}$ europrosthetic devices and Brain Machine Interfaces (BMIs) are increasingly playing a vital role in helping patients with severe motor disorders achieve a better lifestyle by enabling direct interface to the central nervous system at various levels. Recording the activity of cortical neurons with microelectrode arrays was shown to be essential to quantify their degree of involvement in encoding movement parameters, thereby permitting decoding of the neural signals to take place to control artificial limbs [1].

The ability to implant devices into living neural tissue is limited, in part, by volume and power dissipation constraints set to ensure neighboring tissue and nerves are not damaged. For implanted microelectrode arrays, the power required to wirelessly transmit raw data to extra-cranial processing units is prohibitively large. Likewise, the hardware required to perform full neural data analysis is too complex to be implemented within an implanted system. Data compression before transmission is an attractive alternative, if it can be preformed with limited hardware. The Discrete wavelet transform (DWT) was shown to be a very effective signal processing tool for compressing neural data [2].

Fig. 1 shows the block diagram of proposed implantable neural data compression system. Multiple channels of data are captured by the implanted probe and are converted from analog to digital signals. This $\mathrm{A} / \mathrm{D}$ conversion is followed by a spatial filter to remove redundancy across channels, if needed. These channels are then multiplexed and discrete wavelet transformation is applied. Run Length Encoding (RLE) follows the transform and finally the data is transmitted outside the body. The non-zero DWT coefficients give a sparse representation of the signal and

Manuscript received February 16, 2007. This work was supported by NIH grant \# NS047516-01A2

The authors are with the ECE Dept. at Michigan State University, East Lansing, MI 48824 USA. Correspondence should be sent to koweiss@msu.edu. greatly reduce the power required to upload data. This paper focuses on the area-power efficient hardware design of the DWT block, discusses the design decisions, tradeoffs and results.

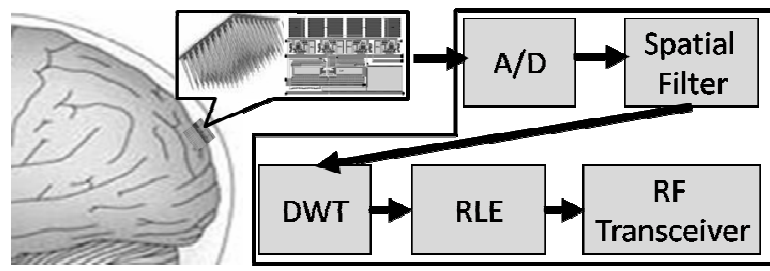

Fig. 1. Block diagram of the proposed implantable neural data compression system with required components.

\section{THEORY}

With modern neuroprosthetics devices, a multi-channel, multi-level DWT implementation is preferred because microelectrode arrays sample multiple data channels simultaneously and multiple decomposition levels improve signal reconstruction accuracy. Thus, area and power efficient hardware that can perform multi-channel, multilevel DWT in real time is highly desirable. In contrast to traditional DWT applications, neuroprosthetics can afford relatively longer computation intervals, up to $40 \mu \mathrm{sec}$ [2], permitting hardware to prioritize power and area efficiency over speed.

In prior work, we have identified an optimal architecture for multi-channel, multi-level DWT in neuroprosthetic applications [3]. The approach relies on the hardwareefficient integer lifting factorization scheme and the 'symmlet' family of wavelet basis that has been shown to provide a near optimal compression of neural signals [2]. Table I gives coefficient values for the 'symmlet 4' filters.

TABLE I. COEFFICIENT VALUES FOR SYMMLET 4 WAVELET FILTERS
\begin{tabular}{|c|c|c|c|c|}
\hline Lag & $\mathbf{z}^{\mathbf{0}}$ & $\mathbf{z}^{-1}$ & $\mathbf{z}^{-2}$ & $\mathbf{z}^{-3}$ \\
\hline Coeff. & -0.076 & -0.030 & 0.498 & 0.804 \\
\hline Lag & $\mathbf{z}^{-4}$ & $\mathbf{z}^{-5}$ & $\mathbf{z}^{-6}$ & $\mathbf{z}^{-7}$ \\
\hline Coeff. & 0.298 & -0.099 & -0.013 & 0.032 \\
\hline
\end{tabular}

Motivation to use the lifting scheme for DWT comes from its ability to reduce the number of computations required as compared to the conventional convolution based filtering. For the 'symmlet 4' bases, if scaling is ignored, convolution based filtering requires 14 multiplications and as many additions, whereas, using lifting the same filtering requires only 8 multiplications and 8 additions. Fig. 2 shows the data flow diagram of lifting-based DWT using the symmlet 4 bases. If $h$ and $f$ are two sequential input samples from the same data channel, $a$ and $d$ are approximation and 
detail results of one level DWT, and $P, Q$, and $R$ are intermediate results, the five filtering steps in this flow can be expressed by

$$
\begin{aligned}
& P_{0}=h_{0}+B_{0} f_{0} \\
& Q_{-1}=f_{-1}+B_{1} P_{0}+B_{2} P_{-1} \\
& R_{-1}=P_{-1}+B_{3} Q_{-1}+B_{4} Q_{-2} \\
& a_{-1}=Q_{-1}+B_{5} R_{-1}+B_{6} R_{-2} \\
& d_{-2}=R_{-2}+B_{7} a_{-1}
\end{aligned}
$$

where $B_{0}-B_{7}$ are the eight constant filter coefficients given in Table II and subscripts of the other variables represent the time sample, 0 is the current sample, -1 is the previous sample, and so on.

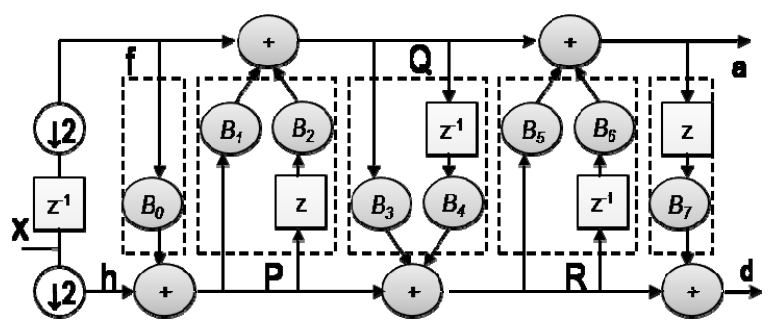

Fig. 2. Data flow diagram for Lifting DWT using symmlet 4 bases (see text for symbol definitions).

TABLE II. COEFFICIENT VALUES FOR LIFTING SYMMLET 4 FILTERS

\begin{tabular}{|l|c|r|r|r|}
\hline Coeff. & $\mathbf{B}_{\mathbf{0}}$ & $\mathbf{B}_{\mathbf{1}}$ & $\mathbf{B}_{\mathbf{2}}$ & $\mathbf{B}_{\mathbf{3}}$ \\
\hline Value & 0.391 & -0.124 & -0.339 & -1.420 \\
\hline Coeff. & $\mathbf{B}_{\mathbf{4}}$ & $\mathbf{B}_{\mathbf{5}}$ & $\mathbf{B}_{\mathbf{6}}$ & $\mathbf{B}_{\mathbf{7}}$ \\
\hline Value & 0.162 & 0.431 & 0.146 & -1.049 \\
\hline
\end{tabular}

Derived from our prior system-level analysis [3], we have found that the power-area product can be minimized by a hardware architecture that sequentially evaluates the DWT of multi-channel data in real time. Based on this analysis, we have chosen 10-bits data and 5-bit coefficients (including sign bit) for our hardware implementation. Fig. 3 describes the DWT architecture resulting from our circuit-level design. It is composed of a customized computation core (CC), a digital controller, and five memory modules for incoming data, filter coefficients, intermediate $\mathrm{CC}$ products, and intermediate values necessary to sequentially process multiple levels and channels. Sequential data samples (inputs $h$ and $f$ ) from a given channel are processed in pairs to generate the approximation and detail DWT coefficients (outputs $a$ and $d$ ). To achieve system-level goals of power and area efficiency, each of these architectural blocks has been customized at the circuit level to minimize transistor count and eliminate unnecessary output transitions.

\section{COMPUTATION CORE}

Analysis of the 'symmlet4' lifting factorization and resulting equations in (1) shows a noticeable computation regularity; all arithmetic operations can be expressed in the general form of $W=X+B_{i} Y+B_{j} Z$. This regularity can be exploited to minimize hardware by implementing a single computation core (CC) that sequentially executes all computational steps in (1). Sequential reuse of the same hardware significantly reduces chip real estate requirements without impacting performance in this low bandwidth application [3]. The merits of using two's complement arithmetic versus sign-magnitude arithmetic were considered. We have implemented several combinations of adders and multipliers using sign-magnitude and two's complement representation and found that it is most efficient to handle multiplication in sign-magnitude form while additions are performed in two's complement. As shown in Fig. 4, the computation core can be implemented using two multipliers and a three-term adder formed by cascading two two-term adders. Using this $\mathrm{CC}$ to sequentially execute the steps in (1) requires five cycles to compute results for one sample pair.

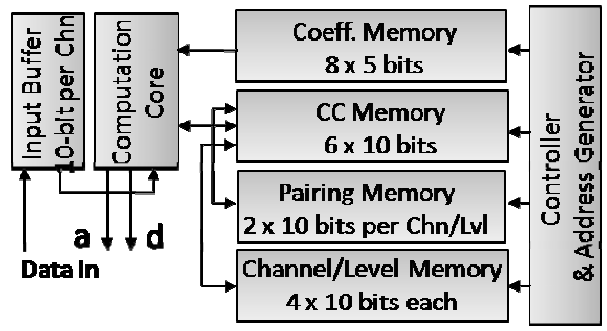

Fig. 3. System diagram for sequential calculation of DWT.

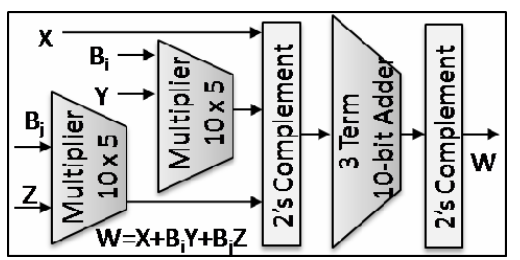

Fig. 4. Computation core architecture for lifting DWT to compute (1).

\section{A. Adders}

Ripple carry, carry save and carry look-ahead adders were analyzed for this DWT implementation. Because delay does not pose a bottleneck and area and power are much more important, the lower transistor count required by ripple carry adder was favored. Ripple carry adder performance depends largely upon structure of its individual full-adder cells, whose properties can vary widely to match different applications. Comparative analysis of several full adder structures was performed at the circuit level to take into account transistor count, activity factor, and dynamic power due to parasitics. Based on these results, the pass gate logic 1-bit full adder presented in [4] and shown in Fig. 5 was chosen to best suit our DWT application.

Unlike some adder cells with slightly lower transistor counts, this cell has no internal direct path between power and ground, eliminating short circuit current during transistor switching. Furthermore, the use of transmission gates limits the output voltage swing and reduces dynamic power consumption without compromising overall speed performance or reliability. On $0.18 \mu \mathrm{m}$ CMOS process, this 16 transistor cell was designed to dissipate $0.4 \mu \mathrm{W}$ power and occupy $41.5 \mu \mathrm{m}^{2}$. 


\section{B. Multipliers}

The $\mathrm{CC}$ requires two multipliers to perform $10 \times 5$ operations. Both Booth and array multiplier structures were optimized to the specific bit resolutions of our DWT application and compared for area and power. The Booth multiplier consists of recoders, partial product generators, 3:2 and 2:1 compressors and a final adder. The recoders and partial product generators were optimized for low power [4]. Two different recoders and partial product generators were considered both requiring 18 transistors each. Two partial product generator, NPR3a and NPR3b, were tailored to this application, and it was determined that, while power consumption is similar, NPR3b needs slightly fewer transistors (14 vs. 16) and is preferred. The 3:2 and 2:1 compressors are full and half adders respectively. The array multiplier ANDs every bit of the multiplier with every bit of the multiplicand to create partial products. A Wallace tree structure was implemented to compress the partial products. Both Booth and array multipliers require an adder at the final stage. In comparison, a CC design using a Booth multiplier was shown to occupy $16 \%$ more area than a $\mathrm{CC}$ design with an array multiplier. Table III gives area, power and delay measurements of the custom designed adder and array multiplier sub-modules selected for use in our DWT CC.

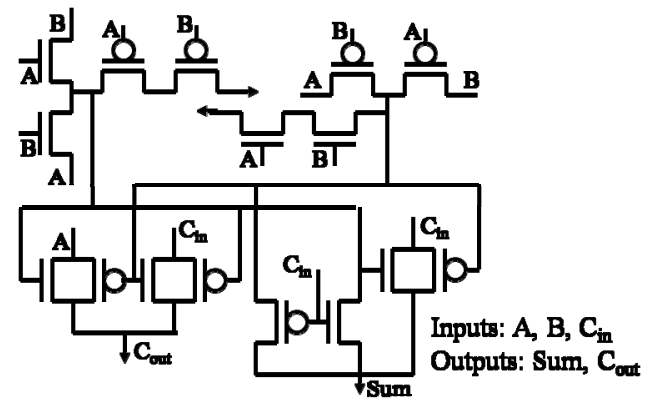

Fig. 5. Single bit full adder cell used in the CC adders [3].

TABLE III. CC TRANSISTOR COUNTS AND WORST CASE VALUES

\begin{tabular}{|l|l|l|l|l|}
\hline Sub-module & \# of TX & $\operatorname{Area}\left(\boldsymbol{\mu m}^{2}\right)$ & Power $(\boldsymbol{\mu W})$ & Delay (ns) \\
\hline Adder & 160 & 418 & 3.834 & 0.404 \\
\hline Multiplier & 733 & 2858 & 42.76 & 4.12 \\
\hline
\end{tabular}

\section{MEMORY MOdULES}

\section{A. Multi-channel/level memory module}

Neuroprosthetic applications generally depend upon multiple data streams coming from an array of microelectrodes. Thus, our DWT system has been designed to compress data from multiple channels simultaneously using multi-level decomposition in real time. Analysis of our DWT implementation indicates that there is sufficient bandwidth within a single computation core to process well over 100 data channels sequentially [3]. Sequential processing significantly reduces computational hardware demand; however, the intermediate values, or 'state', of the current channel needs to be stored in memory so they are available when the $\mathrm{CC}$ later processes the next sample from this channel. Similarly, memory is needed to store intermediate values while switching between different levels. The memory block required to hold these intermediate values is called the channel/level memory. The values that need to be stored and made available to process future samples are defined by (1). For each level and channel (beyond one), the lifting architecture requires five 10-bit values, $a_{0}, f_{0}, P_{0}, Q_{0}$ and $R o$, to be saved. Every level and every channel requires a corresponding 40-bit memory register. In our 32-channel, 4-level design, this amounts to 128 registers. The channel/level memory was implemented using an array of standard 6T SRAM cells. For a large number of channels and levels, the channel/level memory module was found to dominate the power and area of the overall DWT system.

Because DWT operates on data pairs, there is an idle cycle between each computation wherein the first sample of the pair is acquired. Thus, input data buffer of size equal to the number of channels is required to store input samples during the idle cycles. This idle state can be used to compute all results for higher (beyond one) levels of decomposition, where the input data is available from previous computations.

\section{B. Coefficient, pairing and computation core memory modules}

Due to the sequential reuse of CC hardware, intermediate results from the evaluation of (1) must be stored in computation core memory for use in subsequent filter steps. A total of six 10-bit registers are required to hold intermediate values during a given computation cycle. All the registers have parallel and serial load capability. When performing calculations for higher levels a pairing memory is used to store input values. This memory must contain two 10-bit values for every channel and level except the highest level. This memory block must write only one 10-bit value but be able to read all 20 bits stored for a channel/level. Due to this read/write structure this memory block was built separate from the channel/level memory and implemented as two separate blocks of SRAM controlled by the same address decoder circuitry. An enable bit for each block is used to select between them. A separate coefficient memory block is required to store the eight 5-bit filter coefficients. Because this data is static, it can be hardwired and effectively implemented as a ROM.

\section{Power saving strategies for memory}

To reduce power consumption in the memory, a divided bit line structure was used [5] to reduce the overall bit line capacitance that is charged/discharged during read and write cycles. The bit lines were divided into sub bit lines that connect to only eight SRAM cells. Some extra logic was contained in the decoder to control access to these sub bit lines. In addition, the access transistor in the six-transistor SRAM cell is only turned on once during a read cycle to reduce switching power. With reduced bit line capacitance, the SRAM could be implemented without a sense amplifier, which eliminates the need to access both sides of the SRAM 
cell and reduces bit line charging/discharging currents. This also helps to reduce the overall memory power consumption.

\section{Controller Design}

The main functions of the controller are to direct overall system operation and route data between DWT circuit blocks at the proper time. Two different controller options were considered, an instruction based microprocessor and a state machine based controller. The repetitive and sequential nature of the required controller functions warrants the use of a state machine based design. Three different phases of controller operation containing a total of eight clock cycles (per channel per level) are required to process each sample. One read cycle is followed by five calculation cycles for the $\mathrm{CC}$ and two write cycles. The time period allowed to complete these cycles dictates the clocking frequency of the circuit. For 32 data channels and a sampling frequency of 25 $\mathrm{kHz}$, a clock frequency of $6.4 \mathrm{MHz}$ is required. Note that increase in the number of levels has no effect on the clocking as computation of higher level results can be completed in cycles not used by lower levels as can be seen in Fig. 6 .

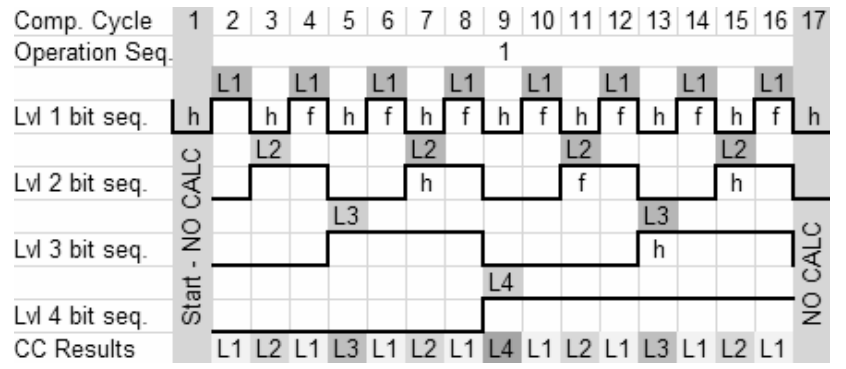

Fig. 6. Per channel activity of $\mathrm{CC}$ at 4 levels of decomposition.

For the state machine to work correctly, the number of incoming channels and the required levels of DWT should be known beforehand. In our implementation the incoming data from multiple channels is assumed to be multiplexed onto a single data line, so the system accepts input from one data bus and outputs data on another. The state machine controller depends on a counter to keep track of its states and control the DWT block at different levels of system hierarchy. The three least significant bits control the eight phases of operation of $\mathrm{CC}$ and memory for a single data pair irrespective of channel or level being processed. For a 32 channel, 4 level system, the next six bits control the channel being processed. This section also directs the input stream to the input buffer or the computation core. The most significant channel bit in conjunction with the three level bits are used to represent the level being processed where each bit represents a separate level. A level 1 result is computed once a pair of data samples is received; a level 2 result is computed when two level one results are available and so on. By using 4 bits to represent the levels we are able to recognize idle cycles for use in calculating higher level results and also to manage storing the approximation coefficient.

Fig. 7 describes the part of the state machine controlled by the three least significant bits of the counter, i.e. the complex data movement within the $\mathrm{CC}$ memory and its interaction with other memory blocks. The top row mentions register names where $\mathrm{X}, \mathrm{Y}$ and $\mathrm{Z}$ are registers connected to respective inputs of the $\mathrm{CC}$ while $\mathrm{M} 1, \mathrm{M} 2$ and $\mathrm{M} 3$ are intermediate memory registers. Values are read into the $\mathrm{CC}$ memory in the first cycle, followed by five cycles of calculations and data shifts. The last computation cycle produces two results; the detail coefficient is transmitted out while the approximation coefficient is transmitted or retained based on the level being calculated. The 4 values in CC memory, $f_{0}, P_{0}, Q_{0}$ and $R_{0}$ are stored in channel/level memory to be reused for higher level calculations.

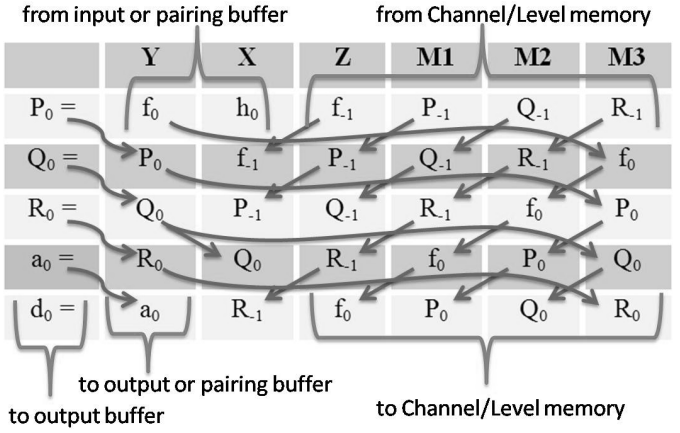

Fig. 7. Data movement in $\mathrm{CC}$ memory during the five calculation cycles within a computation cycle (one per sample per level).

\section{ANALYSIS AND RESULTS}

All of the DWT circuit blocks were custom designed and laid out in $0.18 \mu \mathrm{m}$ CMOS. Table IV lists number of transistors required for each module. The corresponding chip area required by each module, including routing, is also shown. The dominance of channel/level memory over other modules is evident, where increasing the number of channels by a factor of four (from 8 to 32 ) results in roughly a $300 \%$ increase in both the number of transistors and the area consumption (312\% number of transistors, $296 \%$ area). Our model 32-channel, 4-level DWT implementation, requires $\sim 42 \mathrm{k}$ transistors and occupies roughly $400 \mu \mathrm{m} \times 400 \mu \mathrm{m}$.

TABLE IV. TRANSISTOR COUNT AND AREA FOR HARDWARE MODULES

\begin{tabular}{|l|l|l|}
\hline \multicolumn{1}{|c|}{ Module } & No. of Tx & Area $\left(\boldsymbol{\mu m}^{\mathbf{2}}\right)$ \\
\hline Controller & 517 & 3834 \\
\hline Comp Core & 1786 & 13828 \\
\hline Comp Core Memory & 396 & 1775 \\
\hline Coefficient Memory & 264 & 1114 \\
\hline Pairing Memory per channel/level & $120^{*}$ & Varies \\
\hline Input Buffer per channel & $60^{*}$ & Varies \\
\hline Per Channel or Level Memory & $240^{*}$ & Varies \\
\hline Complete DWT system: 8 Chn, 4 Lvl & 13465 & 55248 \\
\hline Complete DWT system: 32 Chn, 4 Lvl & 41943 & 163377 \\
\hline
\end{tabular}

*Counts exclude transistors for address decoding circuitry

The DWT circuit outputs interleaved approximate and detail transform coefficients that give a sparse representation of the original neural signal. Coefficient values below a specific threshold can be set to zero to compress the results into a smaller number of bytes. The non-zero coefficients can then be encoded using a lossless encoding scheme and transmitted to the extra-cranial or extra-cutaneous processing 
units. Choosing the value of the threshold provides a tradeoff between signal integrity and compression ratio.

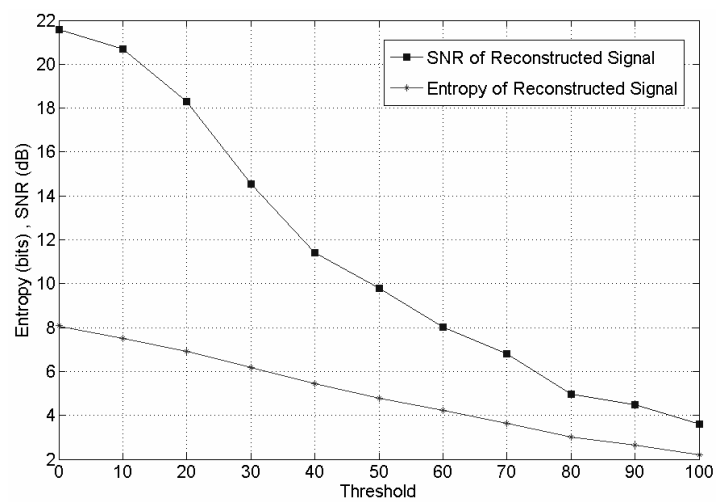

Fig. 8. SNR in $\mathrm{dB}$ and Entropy in bits per symbol as a function of threshold value for the neural data set used in our experiments.

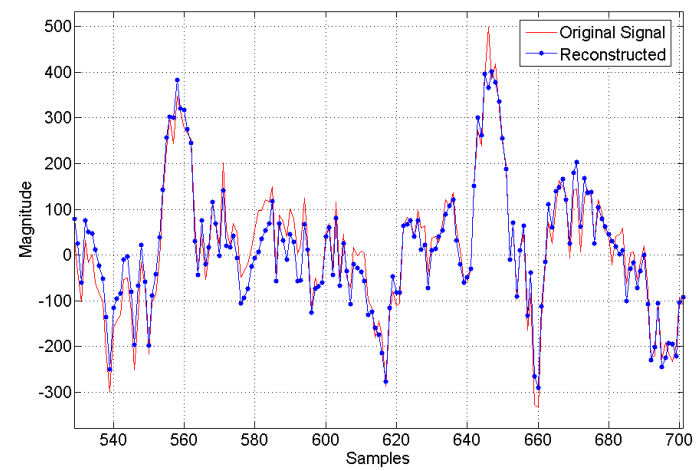

Fig. 9. Original and reconstructed signals for Threshold $=50, \mathrm{SNR}=$ $9.78 \mathrm{~dB}$ and Entropy $=4.78$ bits per symbol at 4 levels of dernmonecition writh 10 hite data and 5 hite mofficiante

To test our DWT circuit implementation on real neural data, a linear-step analog to digital converter was used to convert experimentally obtained neural data into a stream of 10-bit digital values. The data was then processed through the DWT system and results were stored for analysis. In one test, the stored transform coefficients were used to reconstruct the neural signal, and this result was compared to the original signal to measure the quality of reconstruction. This analysis was performed for several different zeroing threshold values to evaluate signal quality verses the amount of compression obtained. The final performance metrics can be defined in terms of the signal to noise ratio (SNR), Shannon's entropy and the assigned threshold. SNR is a ratio of the signal power to the noise power. Here, noise is the difference between the original signal and the reconstructed signal, which has two components: the quantization noise, and the lossy-compression noise resulting from the thresholding operation. The mathematical representation of SNR in decibels $(\mathrm{dB})$ is given by

$$
S N R=10 \log _{10} \frac{\text { signal_energy }}{\text { noise_energy }}
$$

Shannon's entropy is a measure of uncertainty associated with a random signal and can be interpreted as the average minimum message length, represented in bits, which must be transmitted to communicate the exact value of the signal. Entropy gives the theoretical limit to the best possible lossless data compression for any communication. Entropy is mathematically represented as

$$
H(X)=-\sum_{i=1}^{N} p\left(x_{i}\right) \log _{2} p\left(x_{i}\right)
$$

where $N$ is the total number of possible values (also called symbols in information theory literature) and $p\left(x_{i}\right)$ is the probability of occurrence of the $i^{\text {th }}$ value.

In general, increasing the zeroing threshold improves the amount of compression achieved (decreasing entropy) but degrades SNR. For neural data processed through our DWT circuit, Fig. 8 shows the SNR and entropy as the zeroing threshold increases and confirms the anticipated tradeoff. Because of this direct tradeoff between SNR and compression ratio, the zeroing threshold must be chosen to match application requirements; if bandwidth limitations are not severe, a low threshold could maximize signal reconstruction, or if near perfect reconstruction is not needed, a high threshold could reduce power for data transmission. Fig. 9 shows the original and reconstructed signals for threshold values of 50. At this value, an entropy of 4.78 bits per symbol and an SNR of $9.78 \mathrm{~dB}$ were obtained.

\section{CONCLUSION}

We have proposed a system designed to pseudosimultaneously perform multi level DWT for multiple channels of recorded neural signals, thereby enabling very high data compression rates while maintaining high signal fidelity. Our implementation in $0.18 \mu \mathrm{m}$ CMOS requires only $0.16 \mathrm{~mm}^{2}$ of area to process 32 channels of data at 4 levels of DWT in real time. The small size and low power consumption of the system makes it highly suitable for implantable high-density microelectrode array devices.

\section{REFERENCES}

[1] M. Nicolelis, "Actions from Thoughts," Nature, vol. (409): 403-407, Jan. 2001

[2] K. Oweiss, "A Systems Approach for Data Compression and Latency Reduction in Cortically Controlled Brain Machine Interfaces," IEEE Tran. on Biomed. Eng., vol. 53, no. 7, pp. 1364-1377, 2006.

[3] K. Oweiss, A. Mason, Y. Suhail, K. Thomson, and A. Kamboh "A Scalable Wavelet Transform VLSI Architecture for Real-Time Signal Processing in High-Density Intra-Cortical Implants," IEEE Transactions on Circuits and Systems, in press

[4] A. Shams, T. K. Darwish, and M. A. Bayoumi, "Performance analysis of low-power 1-bit CMOS full adder cells," IEEE Trans. VLSI systems, vol. 10, no. 1, pp. 20-19, Feb 2002.

[5] Z. Huang, "High-Level Optimization Techniques for Low-Power Multiplier Design,” PhD dissertation, UCLA, June 2003.

[6] B.-D. Yang, L.-S. Kim, "A low power SRAM using hierarchical bit line and local sense amplifiers," IEEE Journal of Solid State Circuits, vol. 40, no. 6, pp. 1366-1376, June 2005. 